\title{
A Look into the Future: Cyber-aggression as the Problem of Interaction in Space Exploration
}

\section{Dmytro Dzvinchuk}

\author{
Doctor of Philosophical Sciences, Professor, \\ Ivano-Frankivsk National Technical University of Oil and Gas \\ (Ivano-Frankivsk, Ukraine) \\ E-mail: dzvin56@ukr.net \\ ORCID: 0000-0002-6391-3822
}

\section{Oleksandra Kachmar}

\author{
Doctor of Philosophical Sciences, Assistant Professor, \\ Vasyl Stefanyk Precarpathian National University \\ (Ivano-Frankivsk, Ukraine) \\ E-mail: Ooleksandra75@ukr.net \\ ORCID: 0000-0002-2002-4603
}

\begin{abstract}
In the article, the authors broadened on the definition and concept of cyber-aggression. The authors have carried out their inquiry on the cyber-aggression in terms of the possibility of broadening of human potential by using the high-tech devices. Such inquiry was built upon the "The theory of war and peace" by Oleg Bazaluk. The authors came to the conclusion that cyber-aggression appears to be a typical form of struggle for existence, which is inherent in man, the state and civilization. Cyber-aggression, considered as a "natural way of human intelligent activity broadening by using the high-tech devices" provides the following: a) the transformation of civilization into a planetary force; b) exploration of cislunar and deep outer space; c) Universe-wide competition. The authors proposed a behavior model, specially developed for the contacts with extraterrestrial civilizations, which stipulates the transparency of intentions and proves: a) that terrestrial civilization's cyber-aggression is a method of space exploration, rather than a desire for war and violence; b) that the Earthly civilization's technologies are determined by its cultural history and manifest peace and benevolence.
\end{abstract}

Keywords: cyber-aggression, space exploration, extraterrestrial civilizations, theory of war and peace, interstate relations

Received: February 5, 2018; accepted: April 28, 2018

Philosophy and Cosmology, Volume 21, 2018: 54-60

DOI: 10.29202/phil-cosm/21/6

\footnotetext{
(C) Dzvinchuk, Dmytro, 2018

(C) Kachmar, Oleksandra, 2018
} 


\section{Introduction}

In the recent article "Cyber-Aggression: Definition and Concept of Cyberbullying", Dr Dorothy Wunmi Grigg showed that the concept of cyber-aggression is "used to describe a wide range of behaviours other than cyberbullying" [Grigg, 2010: 143]. In Grigg's opinion, cyberaggression includes "a broader definition in line with the current trend of a range of behaviours that are common with internet and mobile phone usage" [Grigg, 2010].

Peter Smith in the particular chapter "Cyberbullying and Cyber Aggression" outlines the history of research in cyberbullying and cyber-aggression and some major publications. He observes types of cyber-aggression and cyberbullying, and features that tend to make it distinctive from "traditional" (noncyber) forms; and considers essential issues that arise. Smith found out a number of facts crucial for cyberbullying and cyber-aggression: "incidence, who does it and where it happens, age and gender differences, other predictors of involvement in cyberbullying, the impact of cyberbullying, coping strategies, prevention and intervention procedures, and concluding with implications for research and practice" [Smith, 2012].

The article "The Association Between Cyber Victimization and Subsequent Cyber Aggression: The Moderating Effect of Peer Rejection" by Michelle F. Wright and Yan Li observes the relationship between cyber victimization and the subsequent cyber aggression of adolescents $6^{\text {th }}, 7^{\text {th }}$, and $8^{\text {th }}$-graders [Wright $\&$ Li, 2013].

Analyzing previous works on cyber-aggression, Matthew W. Savage and Robert S. Tokunaga attempt to utilize general aggression theory to contribute to a better theoretical understanding of the confluence of inputs that goes into the decision-making involving cyberbullying perpetration [Savage \& Tokunaga, 2013].

The inquiries analysis in this field showed that the manifestations of cyber-aggression are mainly researched in adolescents: incidence, who does it and where it happens, age and gender differences, etc. We consider the all achieved in this field results to be an important stage in the study of cyber-aggression, but our research provides a look into the future. In the article, we are going to broaden a comprehension of cyber-aggression phenomenon and to observe its manifestations in other areas such as war and peace, interstate relations, and human space exploration.

\section{Aggression in the theory of war and peace}

In the article "Moving toward a Theory: Testing an Integrated Model of Cyberbullying Perpetration, Aggression, Social Skills, and Internet Self-efficacy" authors utilized general aggression theory to systematize modern consideration of cyberbullying and cyber-aggression [Savage \& Tokunaga, 2013]. This makes sense because cyberbullying and cyber-aggression are manifestations of the psyche and, can be explained by general aggression theory. However, for the basis of our research on cyber-aggression, we propose "The theory of war and peace", proposed by Oleg Bazaluk in 2015 [Bazaluk \& Blazhevych, 2016; Bazaluk, 2017; Bazaluk \& Svyrydenko, 2017]. The following features of the theory of war and peace, crucial for cyberaggression consideration specified our decision.

1. This theory dates back to Plato's and Aristotle's studies and the majority of well known key philosophers made a contribution to it [Fatkhutdinov \& Bazaluk, 2018].

2. It involves modern neuroscience achievements, systematized by Neurophilosophy [Bazaluk, 2017].

3. The theory includes the main provisions of general aggression theory and essentially broadens its theoretical justification and practical use [Bazaluk, 2017]. 
4. The theory of war and peace explains the peculiarities of states evolution and interstate relations development, the reasons for wars and peace in the history of culture [Bazaluk \& Blazhevych, 2016; Bazaluk \& Svyrydenko, 2017].

5. The theory of war and peace was also created in order to explain the peculiarities of possible communication with extraterrestrial civilizations [Bazaluk, 2017].

Consequently, "The theory of war and peace" by Bazaluk provides theoretical and practical conditions for an essential extension of the definition and concept of cyber-aggression. Basing on this theory, we are going to prove that cyber-aggression is natural not only for the planetary and cosmic human activity but is the manifestation of any other extraterrestrial civilization. Accordingly, cooperating with extraterrestrial civilizations mankind should be prepared and able to identify the possible manifestations of cyberbullying and cyber-aggression. This means to be able to distinguish within the extraterrestrial cyber-aggression the elements of violent aggression, and natural aggression, as a manifestation of the rational activity provided in unknown or not accepted by earthly civilization way.

\section{The theory of war and peace in interstate relations}

The practical application of the Bazaluk's "theory of war and peace" in interstate relations appears as the second crucial point in order to explore the concept of cyber-aggression in various models of interactions between extraterrestrial civilizations. In Bazaluk's "theory of war and peace", aggression phenomenon is considered as 1) a regular function of the brain, due to the peculiarities of its structure; 2) as a result of the informational, communicative and educational technologies impact [Bazaluk, 2017]. Bazaluk writes: "According to Dinesh Bhugra, as a result of 132 pieces of international research, it was found that the median prevalence of schizophrenia was 4.6 per 1,000 persons, and morbid risk of disease was 7.2 per 1,000 persons during a lifetime. If the other pathologies in the neurobiology of the brain are added to these results, then the percentage spread of mentalities that are apt to aggression becomes higher than 1 percent of the total number of a mental space" [Bazaluk, 2017: 77]. With the age and especially under the influence of informational, communicative and educational technologies, the percentage of people prone to manifest an aggression increases from 4 up to $10 \%$ of the whole state population.

A high percentage of people prone to aggression basically explains some peculiarities of the internal and foreign policies of states, as well as the reasons for the manifestation of aggression and peace in interstate relations. Bazaluk proved the provisions of his theory on the instances of the geophilosophy of Europe, in which the policy of the leading European states: Germany, France, Russia and Great Britain plays an important role [Bazaluk, 2017]. We will consider the effect of the mentioned theory on the instance of Slovakia, a small state located in Central Europe. Slovakia became an independent state on 1 January 1993. The domestic and foreign policies of Slovakia are aimed not only at economic integration into the European and world community but also at ensuring their own security. The following issues are relevant for Slovakia:

1. Identities, Democracy, Borders [Rouet \& Ušiak, 2017];

2. Contemporary Global Terrorist Movement [Kosárová \& Ušiak, 2017];

3. Military Recruitment Model for Armed Forces of Small States and Middle Power [Ušiak \& Gorner, 2018];

4. Slovakia's perspective on NATO [Ušiak, 2018].

Slovakian instance shows that different forms of aggression are present not only in the policies of large and high-populated states but small ones as well. Each state is compelled to use 
some forms of aggression in its domestic and foreign policy in order to defend its independence and the identity of its culture. We came to the conclusion that aggression appears as a typical form of struggle for existence, which is inherent in both the single person and the policy of any state. In interstate relations, aggression 1) appears as a necessary condition for the development of intelligent matter; 2) it can be stimulated with informational, communicative and educational technologies; 3 ) aggression is also a way of preserving the identity of a person as well as state and civilization.

\section{Definition and Concept of Cyber-Aggression}

Due to the provided analysis now is possible to clarify the definition and concept of cyberaggression. Main clarification is not related to the aggression as a term (most of the scholars are on the same page about its meaning and sense) it concerns the first part of the concept of cyber-aggression, namely the term "cyber".

The reason why the concept of cyber-aggression is limited to the Internet and mobile phones is not clear. In terms of the Transhumanist Declaration and "transhumanism" as a direction of scientific and philosophical research, the significance of cyber-organism and cyber-culture is much wider. Let us highlight the first and last points of the Transhumanist Declaration 1) "Humanity stands to be profoundly affected by science and technology in the future. We envision the possibility of broadening human potential by overcoming aging, cognitive shortcomings, involuntary suffering, and our confinement to planet Earth;" 2) "We favour allowing individuals wide personal choice over how they enable their lives. This includes use of techniques that may be developed to assist memory, concentration, and mental energy; life extension therapies; reproductive choice technologies; cryonics procedures; and many other possible human modification and enhancement technologies" [The Transhumanist Declaration, 2009]. When we are talking about cyber-organism and its manifestations, we mean the possibility of broadening human potential by using the high-tech devices. And this is not only the Internet and mobile devices; these are informational, communicative and educational technologies that broaden the manifestations of the human brain.

Consequently, the cyber-aggression appears to be a natural manifestation of human activity. This statement is sensible because it is evidenced by the history of culture where we can find the instances of the possibility of broadening human potential by using the high-tech devices. Moreover, if cyber-aggression is considered as a broadening of the activity boundaries based on Bazaluk's "theory of war and peace", we would find that cyber-aggression is not only a natural manifestation of the individual activity but a planetary and even cosmic manifestation as well. Cyber-aggression, as an opportunity to broaden the boundaries of the manifestation of the psyche by using the high-tech devices, is available to any intelligent organism located anywhere in the Universe. Trough cyber-aggression, a sensible organism, manifests its identity and protects it.

\section{Cyber-aggression as a prerequisite for a space exploration}

Consideration of a cyber-aggression, as a broadening of the sensible organism's activity boundaries by using the high-tech devices allows us to provide a series of important revisions.

Firstly, cyber-aggression becomes a necessary condition for the transformation of human activity into a planetary force. Broadening individual and collective capabilities by using the high-tech devices, the society eventually might be united into a planetary force. Being in a permanent state of war and peace, solving problems by force and compromises, society is 
guided by one goal - self-preservation. The reasonable manifestation of the cyber-aggression is the main way to represent and conserve the identity of national cultures. Cyber-aggression is the natural aspiration of the organism, society and civilization to survive in a competing environment, which means to manifest oneself and protect the products of its activity. Cyberaggression is a way to civilization-wide compromise and to resolving the conflicts by planetarywide conscious actions.

Secondly, cyber-aggression, considered as a way of sensible organisms' survival, appears as the driving force that directs planetary force to the exploration of the space and the Universe as well. War and peace within a separate civilization, eventually lead to an understanding of the secondary effects of planetary activities. The results of interstate or state coalitions wars appear meaningless in comparison with space safety. Using cyber-aggression as a struggle-for-existence method, society will come to the necessity of the Earth's security strategy development. The problem of human civilization protection from destructive cosmic phenomena will become prior, thereby emphasizing the minor importance of state and regional security strategies. Society will learn how to use cyber-aggression for daring space exploration projects, rather than for planet-wide conflicts and wars.

Thirdly, the Earth's safety strategy does not proclaim any isolation of the planet from space. The safety of the Earth's civilization is related to its involvement in space processes, on a deeper exploration of the Universe and the technologies development, which allow us to adapt to the space phenomena. Cyber-aggression appears as the important factor of space exploration and the usage of the resources of the Universe. Cyber-aggression phenomenon contributes to the development of the technologies, which are able to provide the involvement of terrestrial civilization in space processes: to broaden its manifestations in space, and to protect these manifestations from the destructive influence of the Universe.

Thus, Cyber-aggression, as a natural extension of the possible human activity boundaries by using the high-tech devices, provides the man's overcoming of the boundaries of the planet and the exploration of the capabilities of near space, and later, the Universe. Roman Oleksenko and Lidia Fedorova in the paper "Homo Economicus as the Basis of "Asgardia" Nation State in Space: Perspective of Educational Technologies" proposed a new interpretation of the concept of Homo economicus, in which cyber-aggression is a necessary condition for human space exploration. Oleksenko and Fedorova proposed to consider the concept of Homo economicus as the cultural ideal for educational technologies when creating the "Asgardia" nation state in space [Oleksenko \& Fedorova, 2017].

Cyber-aggression forms the basis for the exploration of the Universe resources in order to create the new technologies. Knowledge of the Universe and technologies for its resources exploration appears as the basis of the Earth's civilization safety strategy. The same knowledge might be considered as a starting point in interaction with extraterrestrial civilizations.

\section{Cyberbullying and cyber-aggression in relations with extraterrestrial civilizations}

The exploration of the Universe and its resources usage in the struggle for existence will eventually involve the terrestrial civilization in competition with extraterrestrial civilizations. There are numerous studies on the existence of extraterrestrial civilizations, for instance, "Life in the Universe: The Abundance of Extraterrestrial Civilizations" by James Newsome Pierce [Pierce, 2008]. Moreover, Bernardo Kastrup offers a new paradigm in which the reason and its carriers occupy an important place in the structure of the Universe [Kastrup, 2018]. We consider extraterrestrial civilizations as an obvious fact that terrestrial civilization will face 
in the short term. For this reason, we propose to consider the model of the behaviour of the Earthly and any other civilization in which cyber-aggression appears as a natural manifestation of Universe-wide competition.

The model of interaction with extraterrestrial civilizations proposes to take into account the historical and cultural context, which is highly important for an intelligent matter. Each civilization has its own history and culture of technics and technology. Therefore, technologies and peculiarities of their usage by terrestrial civilization can be considered as cyberbullying and cyber-aggression by any other civilization. Similarly, the Earth's civilization can consider the extraterrestrial technologies as manifestations of cyber-aggression. However, these technologies might be the representation of extraterrestrial civilization's culture and manifest friendship and peace.

For this reason, cyber-aggression in relations with extraterrestrial civilizations should be considered in the context of their history of the culture. It should not be considered as the manifestation of aggressiveness and malevolence due to the history of Earthly culture. Cyberaggression should not be used as a form of equal response. It is necessary to develop a model of relations with extraterrestrial civilizations taking into account the agreements; it is necessary to gain time, in order to identify the culture of extraterrestrial cyber-aggression and the true reasons for its manifestation. Firstly, it is necessary to show that Earth's civilization technologies are no more than an extension of psyche capabilities by using the high-tech devices and they cannot be dangerous for another civilization. It is crucial to be the first to show benevolence, peace and interest in partnership with an extraterrestrial civilization. The first step towards peace and mutual understanding is not the manifestation of weakness, but of the high level of civilization development.

It has to be also considered that Earth civilization technologies, as well as its cyberaggression, might not be safe for extraterrestrial civilization. Intending peace, Earth civilization may unintentionally harm the extraterrestrial civilization by its high technologies and vice versa. The ideals and values of different civilizations may not be similar as well as the actions evaluation scale. Therefore, the relations with extraterrestrial civilizations should be started as tolerantly and transparently as possible, with a clarification of possible mutual technological harm, explanation of the cultural context of dominant ideals and values. The forms of cyberaggression are a manifestation of activity, friendliness and interest in relationships. However, it is necessary to make sure that the interest in the relationship may not cause any harmful unforeseen consequences, which the other side will consider as a threat or violence.

\section{Conclusion}

Interacting with extraterrestrial civilizations, mankind will face manifestations of cyberbullying and cyber-aggression. However, it may not be always considered a destructive action: hostility manifestation, conflicts or reluctance to interact. Mostly the cyber-aggression appears as the natural activity caused by the intention of self-preservation, as well as the expansion of the psyche capabilities by using the high-tech devices. That is why it is important to be prepared for cyber-aggression and be able to identify it correctly. It is a mistake to consider all possible forms of cyber-aggression as cyberbullying or even cyber-victimization. In most cases, it is a natural activity based on the history of culture, technology, ideals and values of civilization.

Earthly civilization has to develop a behavior model, specially for the contacts with extraterrestrial civilizations, which stipulates the transparency of intentions and proves that terrestrial civilization's cyber-aggression is nothing more than a method of space exploration; 
that the Earthly civilization's technologies are determined by its cultural history and manifest peace, benevolence and partnership interest. Contacting with extraterrestrial civilizations, it is important to be the first who discovers the senses of cyber-aggression, in order to enjoy the cooperation and mutual understanding.

\section{[L] References}

Bazaluk, Oleg. The Theory of War and Peace, Geophilosophy of Europe. Newcastle upon Tyne: Cambridge Scholars Publishing, 2017. — 185 p.

Bazaluk, Oleg, and Tamara Blazhevych. The Philosophy of War and Peace. Philosophy and Cosmology, Volume 17, 2016: 12-25.

Bazaluk, Oleg and Denys Svyrydenko. Philosophy of War and Peace: In Search of New European Security Strategy. Anthropological Measurements of Philosophical Research, 12, 2017: 89-99.

Fatkhutdinov, Vasyl H. and Oleg Bazaluk. The Importance of the Brain Neuro-Programming Technologies in National and Regional Security Strategies. Philosophy and Cosmology, Volume 20, 2018: 74-82. DOI: 10.29202/phil-cosm/20/6

Grigg, Dorothy Wunmi. Cyber-Aggression: Definition and Concept of Cyberbullying. Journal of Psychologists and Counsellors in Schools. Volume 20, Issue 2, 2010: 143-156. https:// doi.org/10.1375/ajgc.20.2.143

Kastrup, Bernardo. The Next Paradigm. Future Human Image, Volume 9, 2018:41-51. DOI: $10.29202 /$ fhi $/ 9 / 4$

Kosárová, Dominika and Jaroslav Ušiak: The Role of Identity in the Contemporary Global Terrorist Movement. Politické vedy, Vol. 20, No. 4, 2017: 113-133.

Lamb, Megan. Is Cyber Aggression a New Form of Social Aggression?: An Examination of the Frequency and Motivations for Using These Behaviours. Carleton University, 2015.

Oleksenko, Roman and Lidiia Fedorova. Homo Economicus as the Basis of "Asgardia" Nation State in Space: Perspective of Educational Technologies. Future Human Image, Volume 7: 113-119.

Pierce, James N. Life in the Universe: The Abundance of Extraterrestrial Civilizations. Brown Walker Press, 2008.

Rouet, Gilles and Jaroslav Ušiak. Identities, Democracy, Borders. Politické vedy, Vol. 20, No. 4, 2017: 8-13.

Savage, Matthew W. and Robert S. Tokunaga. Moving toward a Theory: Testing an Integrated Model of Cyberbullying Perpetration, Aggression, Social Skills, and Internet Selfefficacy. Computers in Human Behavior, 71, (353), (2017).

Smith, Peter. Cyberbullying and Cyber Aggression. Handbook of School Violence and School Safety: International Research and Practice. Routledge, 2011.

The Transhumanist Declaration. March 2009. http://humanityplus.org/philosophy/ transhumanist-declaration/

Ušiak, Jaroslav. Slovakia's perspective on NATO. Communist and Post-Communist Studies, Vol. 51. No. 2, 2018. https://doi.org/10.1016/j.postcomstud.2018.04.004.

Ušiak, Jaroslav and Erik Gorner. Military Recruitment Model for Armed Forces of Small States and Middle Powers. Journal UNISCI / Revista UNISCI. 46, 2018: 197-218.

Wright, Michelle F. and Yan Li. The Association between Cyber Victimization and Subsequent Cyber Aggression: The Moderating Effect of Peer Rejection. Journal of Youth and Adolescence. Volume 42, Issue 5, 2013: 662-674. 